\title{
Microvascular and Small-Vessel Disease: An Unrecognized Connection in Women with Modern Coronary Disease
}

\author{
Audry Chacin-Suarez, MD and Jose R. Medina-Inojosa, MD, MSc
}

$\mathbf{I}^{\mathrm{s}}$ SCHEMIC HEART DISEASE and stroke are considered among the leading causes of death and disability in Europe, North America, and many other regions of the world. ${ }^{1,2}$ Although deaths attributable to cardiovascular diseases (CVDs) have steadily decreased over the past few decades, mainly because of major advances in preventative strategies, novel therapeutics, and the increasing availability of revascularization, the most recent United States statistics suggest stagnation in these improvements, especially among women, wherein $\sim 418,665$ excess deaths were associated with CVD. ${ }^{1}$ Therefore, it is crucial that we continue efforts in recognizing the prevalence and mechanism of traditional and novel risk factors that contribute to worsening outcomes in women.

Healthy endothelium is essential for cardiovascular control. In the past, the endothelium was thought to be only a mechanical barrier. Nowadays, we acknowledge that the endothelium orchestrates interactions between vascular tone, growth factors, and thromboregulatory molecules that respond to physical and chemical signals. ${ }^{3}$ Endothelial dysfunction contributes to all the stages of atherosclerosis, shifting the properties of endothelium toward a proliferative, proinflammatory, and prothrombotic state. These pathological alterations are prevalent across an expansive spectrum of cardiovascular risk factors and have been associated with an increased risk of adverse cardiovascular events.

Small vessel disease denotes a group of disorders affecting the performance and structure of the small arteries, arterioles, venules, and capillaries of coronary microvasculature (coronary microvascular dysfunction, CMD) and other organ systems. In the coronary vasculature, decreased epicardial vessels, microvasculature, increased arterial stiffness, altered remodeling, fibrosis and smooth muscle dysfunction, and diffuse atherosclerotic disease have all been observed. ${ }^{4}$

According to the 2019 European Society of Cardiology Chronic Coronary Syndromes and other modern guidelines, CAD is now defined as " a pathological process characterized by atherosclerotic plaque accumulation in the epicardial arteries, whether obstructive or non-obstructive." CMD is a potential cause of ischemia in patients with no obstructive coronary artery disease (INOCA). ${ }^{6}$

As chest pain and dyspnea continue to be the most common presenting symptoms in cardiac patients, no significant obstructive coronary lesions are frequently found in those undergoing cardiac catheterization, and contrary to previous knowledge, prognosis is not necessarily benign. Ample literature has demonstrated that most of myocardial infarctions arise from nonobstructive plaques and many myocardial infarctions are observed during follow-up in patients with nonobstructive disease. ${ }^{7}$

The prevalence of small-vessel disease is higher in women ${ }^{8}$ and is found to be comorbid with diseases that tend to predominantly affect females (e.g., coronary small-vessel disease, cerebral small-vessel disease, pre-eclampsia, pulmonary arterial hypertension, diabetic cardiomyopathy, and some collagen vascular diseases).

In this issue of Journal of Women's Health, Patel et al. ${ }^{9}$ make a strong case for the potential linkage between these multiple diseases that all share common pathophysiological origins and themes: microvascular dysfunction primarily affects women and has potential for accelerating cardiovascular risk.

There are several take-home points from this review. First, the authors underscore the key interaction in which traditional risk factors interplay with endothelial dysfunction and declining estrogen levels to impact CVD. Estrogen is thought to have protective effects, and the postmenopausal estrogen decline mediates the resulting increased sympathetic activation and endothelial dysfunction in the coronary microcirculation. In addition, depression, anxiety, and other forms of mental stress have been associated with an increased risk for coronary heart disease, and these risk factors seem to be more prevalent among women than men.

Previous studies have shown that diabetes appears to lend stronger risk for coronary heart disease in women and impaired glucose rates are higher among women than men. Lack of physician awareness and understanding of pathophysiological differences in heart disease in women may lead to oversight in prevention efforts in diabetes, atherogenic dyslipidemia, and other CVDs.

Second, it is imperative to highlight the clinical importance of endothelial dysfunction in the setting of nonobstructive coronary plaque. ${ }^{10}$ Minimal or no angiographic changes have been noticed in $>50 \%$ of women presenting with symptoms of chest pain, a frequent problem encountered in clinical practice. Similarly, previous invasive flow investigations have suggested that up to two-thirds of patients with

Division of Preventive Cardiology, Department of Cardiovascular Medicine, Mayo Clinic, Rochester, Minnesota, USA. 
nonobstructive coronary atherosclerosis may have CMD. ${ }^{11}$ In a recent evaluation of a large cohort of symptomatic patients undergoing invasive diagnostic coronary angiography for suspected obstructive CAD, $\sim 60 \%$ did not have obstructive CAD. ${ }^{12}$ Furthermore, previous reports have suggested a common association between INOCA and systemic small-vessel disease of the kidney, retina, and cerebral white matter. $^{13}$

Symptomatic patients without identifiable obstructive plaque at times received reassurance that they do not have heart disease, despite signs and symptoms that make them vulnerable for future high-risk cardiac events. An annual MACE rate of $2.5 \%$ has been reported in women with CMD. Recognition that nonobstructive plaque may be associated with future myocardial infarction is imperative, particularly since INOCA appears to be highly prevalent in the general population. At present, no guideline-directed recommendations exist for this population and a knowledge gap about prevention strategies remains.

Lastly and not less important, the authors have underlined a cornerstone piece for prevention and management of these pathological processes, since changes in microcirculation cannot be easily assessed by angiogram. The gold standard for functional assessment of coronary flow is an invasive coronary reactivity test, which has shown to offer additional clinical information in symptomatic women with a normal angiogram; however, it is not widely available. Recent studies have shown that impaired coronary microvascular reactivity predicts major adverse cardiovascular events (MACEs) in women with signs and symptoms of ischemia, even without significant CAD. ${ }^{14}$

Additional noninvasive techniques have emerged to assess coronary microcirculation, myocardial perfusion, and microvascular dysfunction. Cardiac positron emission tomography perfusion imaging can identify microvascular disease by assessing reductions in hyperemic myocardial blood flow, and seems to add clinically relevant prognostic information. Recent investigations suggest that cardiac magnetic resonance and computed tomography may also be growing noninvasive modalities to detect microvascular disease and play a role in the understanding of endothelial dysfunction.

To conclude, Patel et al. ${ }^{9}$ summarize a common, often unrecognized, contributor to the disproportionate CVD burden in women, with shared pathophysiology across multiple organ systems, and highlight the imperative need to close the knowledge gap between optimal prevention and management strategies, on the basis of multidisciplinary collaboration among clinicians caring for women.

\section{References}

1. Virani SS, Alonso A, Benjamin EJ, et al. Heart disease and stroke statistics-2020 update: A report from the American Heart Association. Circulation 2020;141:e139-e596.

2. Roth GA, Johnson C, Abajobir A, et al. Global, regional, and national burden of cardiovascular diseases for 10 causes, 1990 to 2015. J Am Coll Cardiol 2017;70:1-25.
3. Konukoglu D, Uzun H. Endothelial dysfunction and hypertension. Adv Exp Med Biol 2017;956:511-540.

4. Gulati M, Cooper-DeHoff RM, McClure C, et al. Adverse cardiovascular outcomes in women with nonobstructive coronary artery disease: A report from the Women's Ischemia Syndrome Evaluation Study and the St James Women Take Heart Project. Arch Intern Med 2009;169: 843-850.

5. Knuuti J, Wijns W, Saraste A, et al. 2019 ESC guidelines for the diagnosis and management of chronic coronary syndromes. Eur Heart J 2020;41:407-477.

6. Bairey Merz CN, Pepine CJ, Walsh MN, Fleg JL. Ischemia and no obstructive coronary artery disease (INOCA): Developing evidence-based therapies and research agenda for the next decade. Circulation 2017;135:1075-1092.

7. Newby DE, Adamson PD, Berry C, et al. Coronary CT angiography and 5-year risk of myocardial infarction. N Engl J Med 2018;379:924-933.

8. Ford TJ, Rocchiccioli P, Good R, et al. Systemic microvascular dysfunction in microvascular and vasospastic angina. Eur Heart J 2018;39:4086-4097.

9. Patel H, Aggarwal NT, Rao A, et al. Microvascular disease and small-vessel disease-The nexus of multiple diseases of women: A narrative review. J Womens Health 2020;29: 770-779.

10. Boden WE, O'Rourke RA, Teo KK, et al. Optimal medical therapy with or without PCI for stable coronary disease. N Engl J Med 2007;356:1503-1516.

11. Bairey Merz CN, Shaw LJ, Reis SE, et al. Insights from the NHLBI-Sponsored Women's Ischemia Syndrome Evaluation (WISE) Study: Part II: Gender differences in presentation, diagnosis, and outcome with regard to gender-based pathophysiology of atherosclerosis and macrovascular and microvascular coronary disease. J Am Coll Cardiol 2006; 47:S21-S29.

12. Patel MR, Peterson ED, Dai D, et al. Low diagnostic yield of elective coronary angiography. N Engl J Med 2010;362: 886-895.

13. Sun SS, Shiau YC, Tsai SC, Ho YJ, Wang JJ, Kao CH. Cerebral perfusion in patients with syndrome $\mathrm{X}$ : A single photon emission computed tomography study. J Neuroimag 2001;11:148-152.

14. Guerraty MA, Rao HS, Anjan VY, et al. The role of resting myocardial blood flow and myocardial blood flow reserve as a predictor of major adverse cardiovascular outcomes. PLoS One 2020;15:e228931.

Address correspondence to: Jose R. Medina-Inojosa, MD, MSc Division of Preventive Cardiology Department of Cardiovascular Medicine Mayo Clinic 200 First Street $S W$ Rochester, MN 55905 USA

E-mail: medinainojosa.jose@mayo.edu 